\title{
A FRAMEWORK OF PROJECT EVALUATION BASED ON OUTCOME IN LOCAL GOVERNMENT
}

\begin{abstract}
About twenty years ago evaluation was introduced in Japanese local governments. In the beginning this evaluation was considered as a key tool in decentralization reform and the financial crisis, but the aim became ambiguous later. As a result, expectation of evaluation has been fading in spite of call for more rational evaluation. Japanese researchers in policy evaluation pointed out that output indices of projects were not linked to outcome of the program on the upper level in many cases. It is difficult to measure how much each project is effective to the program if it adheres to a quantitative evaluation. The purpose of this study is to show a framework of project evaluation based on outcome of the program and a way to calculate relative evaluation values of projects with respect to the program using the AHP. In this study outcome of the program is represented as a hierarchy of three levels with the program on the top, several viewpoints of the program and several functions of activities of projects to achieve outcome of the program. In this study I defined Contribution degrees of projects to evaluate projects relatively with respect to the program. Relative evaluation values of projects are calculated by this degree. I showed how to calculate relative evaluation values of projects for the program related to gender equality in a local government. The ANP can simultaneously calculate Contribution degrees and relative evaluation values of projects, I did not use it in this study.
\end{abstract}

Keywords: local government, project evaluation, relative evaluation, contribution degree.

\section{Introduction}

About twenty years have passed since evaluation was introduced in Japanese local government administration. This evaluation was introduced as policy evaluation for decentralization reform in the beginning. After that, this evaluation changed to administrative evaluation for a budget cut. Furthermore, it became evaluation with PDCA and KPI like a company under the influence of NPM (new public management). In the inside of these changes, the purpose of the evaluation became ambiguous. As a result, local government administration evaluation has been regarded as a useless work and official workers who draw up evaluation sheets have come to feel "evaluation tiredness".

By the way, local government plans about ten policies every five years or every ten years. After that several programs are planned for each policy, and furthermore, several projects are planned for each program. Actual administrative activities are implemented according to these projects. From the relationship between policies, programs and projects, it turns out that evaluation of projects needs to link to the program. In fact, most Japanese researchers in policy evaluation point out that if projects for a program are separately evaluated and are not relatively evaluated to each other with respect to the program, it is impossible to utilize administrative evaluation for the budgetary process of the following fiscal year or to decide which project should be reduced or expanded.

International Symposium on

1 
The purpose of this study is to show a framework of project evaluation based on outcome of the program and a way to calculate relative evaluation values of projects with respect to the program using the AHP. In this study outcome of the program is represented as a hierarchy of three levels with the program on the top, several viewpoints of the program, and several functions of activities of projects to achieve the program on the bottom. I defined Contribution degree of each project to evaluate projects relatively with respect to the program. The relative evaluation values of projects are calculated by this degree. Furthermore, I showed these computational procedures using the program about gender equality in Chino city, Nagano, Japan. The ANP can simultaneously calculate Contribution degrees and relative evaluation values of projects, I did not use it in this study because of easy understanding of evaluators.

\section{Literature Review}

There are many iteratures about policy evaluation, program evaluation and project evaluation by Japanese researchers. Some of them used the AHP based on the situation that policies, projects and projects construct a hierarchy. The adopted criteria are efficiency, effectiveness, adequacy, fairness and urgency and so on (Tanaka, 2014). Hatry wrote the following in (Hatry, 2007): These objectives normally should be stated in general, not quantitative, terms and should remain reasonably stable. This word is very suggestive, although performance measurement is regarded as a part of project evaluation. As known well, Saaty applied the BOCR model (ANP) to local government (ex. (Saaty, 2005)).

\section{Hypotheses/Objectives}

Most of local government do not evaluate projects relatively under the program, because it is difficult to evaluate projects relatively with respect to the program. So is it, in particular, if local government administrative evaluation is restricted to quantitative evaluation. I am afraid that there often exists this restriction in Japanese administrative evaluation. As a result, the useless administrative evaluation for decision-making causes "evaluation tiredness" to workers as mentioned above. The purpose of this study is to show relative evaluation of projects is easily calculated using the AHP. I also explained the advantage of dividing project evaluation between individual evaluation of itself and relative evaluation with respect to the program.

\section{Research Design/Methodology}

I supposed the following situation to explain this framework. There is a city in Japan, which has several policies. One of them has program $\mathrm{G}$ which has five kinds of projects $\mathrm{P} 1, \ldots$, P5. Furthermore, these projects have already individually evaluated by conventional "quantitative" evaluation method such as target achievement rate as in Table 1.

Table 1

Individual evaluation values for each project

\begin{tabular}{l|ccccc} 
Project & $\mathrm{P} 1$ & $\mathrm{P} 2$ & $\mathrm{P} 3$ & $\mathrm{P} 4$ & $\mathrm{P} 5$ \\
\hline Target growth rate & 0.3 & 0.8 & 0.5 & 0.7 & 0.9
\end{tabular}


ISAHP Article: A Style Guide for Paper Proposals To Be Submitted to the International Symposium on the Analytic Hierarchy Process 2018, Hong Kong, HK.

It follows from Table 1 that Project 5 was evaluated the highest and the organization which worked on this project get high evaluation this fiscal year. Consequently, their motivation will raise in the following fiscal year. On the other hand, administrators of this city need to decide how these projects are in the fiscal year. For instance, they have several options such as expansion, reduction, maintenance of the status quo. This decision is not related directly to individual evaluations of projects, namely, Table 1.

Here, local government need to evaluate these projects relatively with respect to the program G. First, we clarify the direction of outcome of the program using a hierarchy of the AHP. For this we consider viewpoints of the program, which mean aspects of issues to solve. Next, we select functions of projects to realize the ideal society from those viewpoints. These viewpoints and functions should be selected based on results by researchers in the field related to the program. I supposed to obtain the following hierarchy with three viewpoints V1, V2 and V3 and five functions F1, ., F5 (Figure 1).

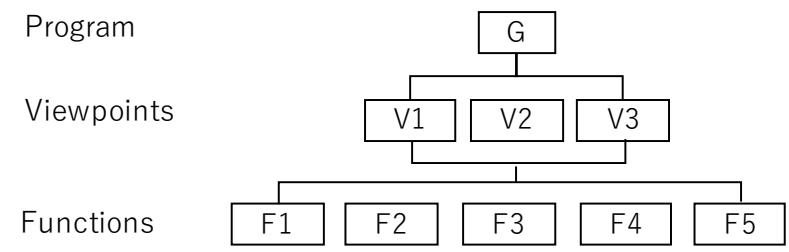

Figure 1 Hierarchy to clarify the direction of outcome

We calculate weights of items of the hierarchy and synthesizing evaluation value of functions Fs with the AHP. I suppose that the following evaluation values of Fs.

Table 2

Synthesizing evaluation values for functions Fs

\begin{tabular}{l|ccccc|c} 
Function & F1 & F2 & F3 & F4 & F5 & Sum \\
\hline Synthesizing evaluation & 0.3 & 0.2 & 0.1 & 0.3 & 0.1 & 1.0
\end{tabular}

Next, we calculate relative evaluation values of projects $\mathrm{P} 1, \ldots, \mathrm{P} 5$ with respect to meaningful functions $\mathrm{F} 1, \ldots, \mathrm{F} 5$ in order to obtain Contribution degree of each project. Because all the projects do not have all the functions, we have to check which functions every project has. I supposed to obtain Figure 2 from this check. Referring to this table, we compare projects with respect to meaning function (Figure 3), for instance only P1 and P2 with respect to $\mathrm{F} 1$.

Table 3

Relationship between Ps and Fs

\begin{tabular}{c|ccccc} 
& F1 & F2 & F3 & F4 & F5 \\
\hline P1 & $\checkmark$ & & $\checkmark$ & $\checkmark$ & $\checkmark$ \\
P2 & & $\checkmark$ & & $\checkmark$ & \\
P3 & $\checkmark$ & $\checkmark$ & & $\checkmark$ & \\
P4 & & $\checkmark$ & $\checkmark$ & & $\checkmark$ \\
P5 & & & $\checkmark$ & $\checkmark$ &
\end{tabular}

International Symposium on the Analytic Hierarchy

Process
Table 4

Weights of Ps with respect to Fs

\begin{tabular}{l|lllll} 
& F1 & F2 & F3 & F4 & F5 \\
\hline P1 & 0.3 & & 0.1 & 0.2 & 0.2 \\
P2 & & 0.2 & & 0.3 & \\
P3 & 0.7 & 0.5 & & 0.3 & \\
P4 & & 0.3 & 0.5 & & 0.8 \\
P5 & & & 0.4 & 0.2 & \\
\hline sum & 1.0 & 1.0 & 1.0 & 1.0 & 1.0
\end{tabular}

3

Hong Kong, HK. July 13 - July 15, 2018 
It is suitable that Table 2 should be obtained by committee members in local government, because paired comparisons to obtain Table 2 are related to the destination of the community. On the other hand, Tables 3 and 4 should be obtained by representatives of all the projects, because they understand projects well. Finally, I combined Table 2 and Table 4 to calculate weights of projects as follows:

$$
\left(\begin{array}{ccccc}
0.3 & 0 & 0.1 & 0.2 & 0.2 \\
0 & 0.2 & 0 & 0.3 & 0 \\
0.7 & 0.5 & 0 & 0.3 & 0 \\
0 & 0.3 & 0.5 & 0 & 0.8 \\
0 & 0 & 0.4 & 0.2 & 0
\end{array}\right)\left(\begin{array}{l}
0.3 \\
0.2 \\
0.1 \\
0.3 \\
0.1
\end{array}\right)=\left(\begin{array}{l}
0.18 \\
0.13 \\
0.40 \\
0.19 \\
0.10
\end{array}\right) .
$$

These weights are one of what we tried to calculate in this study, which were named Contribution degrees (Table 5).

Table 5

Contribution degrees of each project for the program $\mathrm{G}$

\begin{tabular}{l|ccccc} 
Project & P1 & P2 & P3 & P4 & P5 \\
\hline Contribution degree & 0.18 & 0.13 & 0.40 & 0.19 & 0.10
\end{tabular}

To calculate relative evaluation values, I multiplied this Table 5 with Table 1 for every cell. Remember that Table 1 was outside this computational procedure. Table 6 represents relative evaluation value of each project with respect to the program, which are the rest of what we try to calculate in this study.

Table 6

Relative evaluation value of each project with respect to the program $G$

\begin{tabular}{l|ccccc} 
Project & P1 & P2 & P3 & P4 & P5 \\
\hline Relative evaluation & 0.054 & 0.104 & 0.20 & 0.133 & 0.09
\end{tabular}

For instance, it is known that from Project 5 has highest individual evaluation values from Table 1, but low relative evaluation value from Table 4. As the results, Project 5 may be reduced in the following fiscal year.

\section{Data/Model Analysis}

Koizumi and I apply this framework to the project evaluation of the program about gender equality in Chino city, Japan (Koizumi \& Iida, 2018). We set three viewpoints and six functions by Japanese researchers' results in Japanese gender equality issues as follows:

$>$ Viewpoints: (V1) the division of labor by gender (V2) the wage gap by gender (V3) job promotion gap by gender

$>$ Functions: (F1) Place for advice (F2) Opportunity of learning (F3) Environment for access to information (F4) Interaction with specialists (F5) Grasp of actual conditions (F6) Support of promotion in the workplace

\section{Limitations}

First, historically, administrative evaluation is a technique for a budget cut (Yamaya, 2016). The framework in this study does not directly contribute to a budget cut.

International Symposium on the Analytic Hierarchy

Process
Hong Kong, HK. July 13 - July 15, 2018 
ISAHP Article: A Style Guide for Paper Proposals To Be Submitted to the International Symposium on the Analytic Hierarchy Process 2018, Hong Kong, HK.

However, this can contribute to clarification of outcome of the project by not quantitative terms. This is due to a strong point of the AHP. Next, Koizumi and I applied this framework to the program of gender equality for the realistic example. This field has already a lot of results by Japanese researchers and so we can easily decide viewpoints and functions (Figure 1). This has also the advantage that most Japanese people agree with those criteria. However, in general, it is difficult to make viewpoints and functions with which many people agree. This difficulty is like that of a logic model in policy evaluation. Finally, this way is one of group-decision-making and we need to decide a way of paired comparisons as a group. For convenience of participants we may be able to use vote and ICT such as a web site.

\section{Conclusions}

I showed a framework of project evaluation based on outcome of the program in local government administration evaluation using the AHP. This has three kinds of advantages to overcome issues of the existing project evaluation. The first is to clarify viewpoints of outcome of the program for projects, which is the directivity of projects. The second is to divide evaluators' role into two pieces. One is related committee members in local government who are decision makers. The other is people who manage projects such as representatives of NPO. The last is to calculate Contribution degree of each project. It is easy to see that this degree is helpful itself, although this degree was used to calculate relative evaluation of projects in this study. Finally, I used the AHP because of simplicity for evaluators, but at the same time I assumed that all the items are independent mutually. However, those in the same level have dependence between each other. It is a future subject to soak dependence up with the ANP up to the extent that it does not become complicated.

\section{Key References}

Hatry, P.H. (2007). Performance Measurement: Getting Results, 2nd edition. Washington, D.C.: The Urban Institute.

Tanaka, K. (2014). Strategy of local government evaluation (in Japanese). Japan: Toyo Keizai Inc.

Saaty, T.L. (2005) The Encyclicon: A Dictionary of Applications of Decision Making with Dependence and Feedback. Pittsburgh, PA: RWS Publications.

Yamaya, K. (2016). Local government evaluation in decentralization reform and the financial crisis: based on a review of local government administration evaluation for these twenty years (in Japanese). Japanese Journal of Evaluation Studies, 16(1), 31-44. 